\title{
Optimized first-line therapy for stage IV breast cancer brings long-term disease-free survival: a case report and biological analysis
}

\author{
Li Bian ${ }^{1}$, Fengrui Xu ${ }^{1,2}$, Tao Wang ${ }^{1}$, Huiqiang Zhang ${ }^{1}$, Yang Cao ${ }^{3}$, Zefei Jiang ${ }^{1}$ \\ ${ }^{1}$ Department of Breast Oncology, The Fifth Medical Center of Chinese PLA General Hospital, Beijing 100071, China; ${ }^{2}$ Department of Breast \\ Oncology, Academy of Military Medical Sciences, Beijing 100089, China; ${ }^{3}$ Department of Health Medicine, The Fifth Medical Center of Chinese \\ PLA General Hospital, Beijing 100071, China \\ Correspondence to: Tao Wang. Department of Breast Oncology, The Fifth Medical Center of Chinese PLA General Hospital, No. 8 Dongda Street, \\ Fengtai District, Beijing 100071, China. Email: wangtao733073@163.com.
}

\begin{abstract}
For patients with stage IV breast cancer, the purpose of routine treatment is to achieve tumor control and prolonged life without disease progression. But optimized first-line therapy for stage IV breast cancer can bring long-term disease-free survival. We reported a patient with HER2-positive stage IV breast cancer who achieved clinical remission following optimized first-line therapy (albumin-bound paclitaxel plus capecitabine combined with trastuzumab). Meanwhile, the detection of circulating tumor cells (CTC) in combination with PET/CT evaluation showed tumor complete remission, inducing the concept of biological complete remission (bCR). Over 10 years, the patient was followed-up, and she remained in disease free. Circulating tumor DNA (ctDNA) combined with CTC biological analysis was used to elucidate the longterm survival benefits of bCR. Even for stage IV patients, active and reasonable systemic treatment can achieve not only progression-free survival but also disease-free survival.
\end{abstract}

Keywords: Stage IV breast cancer; optimized first-line therapy; disease-free survival; biological analysis; case report

Received: 28 February 2020; Accepted: 24 March 2020; Published: 10 April 2020.

doi: $10.21037 /$ tbcr.2020.04.01

View this article at: http://dx.doi.org/10.21037/tbcr.2020.04.01

\section{Introduction}

Stage IV breast cancer, which refers to those patients who have a distant metastasis at the initial diagnosis, accounts for $5-10 \%$ of breast cancer cases (1). For early breast cancer, after surgery and systemic treatment, patients are considered to be in a state of "disease-free survival" and have a chance to heal. However, unlike early breast cancer patients, those with stage IV disease undergo treatment with the aim of controlling the tumor, thus prolonging life without disease progression, and improving symptoms. Yet, sometimes in clinical therapy, we encounter stage IV breast cancer patients who exhibit complete tumor remission after systemic treatment, and achieve long-term survival.

Patients with stage IV breast cancer who have progressed to distant metastasis can also achieve long-term disease-free survival after initial treatment. The diagnosis and treatment technology in breast cancer have continuously developed over the past decade, including the iteration of NGS, ctDNA, and CTC detection technology, which introduced breast cancer diagnosis and treatment into the era of precision medicine.

Herein, we report a patient with HER2-positivestage IV breast cancer who achieved clinical remission after six cycles of optimized first-line treatment with albumin-bound paclitaxel plus capecitabine combined with trastuzumab. CTC detection in combination with PET/CT evaluation showed tumor complete remission, inducing the concept of biological complete remission (bCR) (2).

This article reports a combination of ctDNA and CTC biological analysis techniques to elucidate the long- 


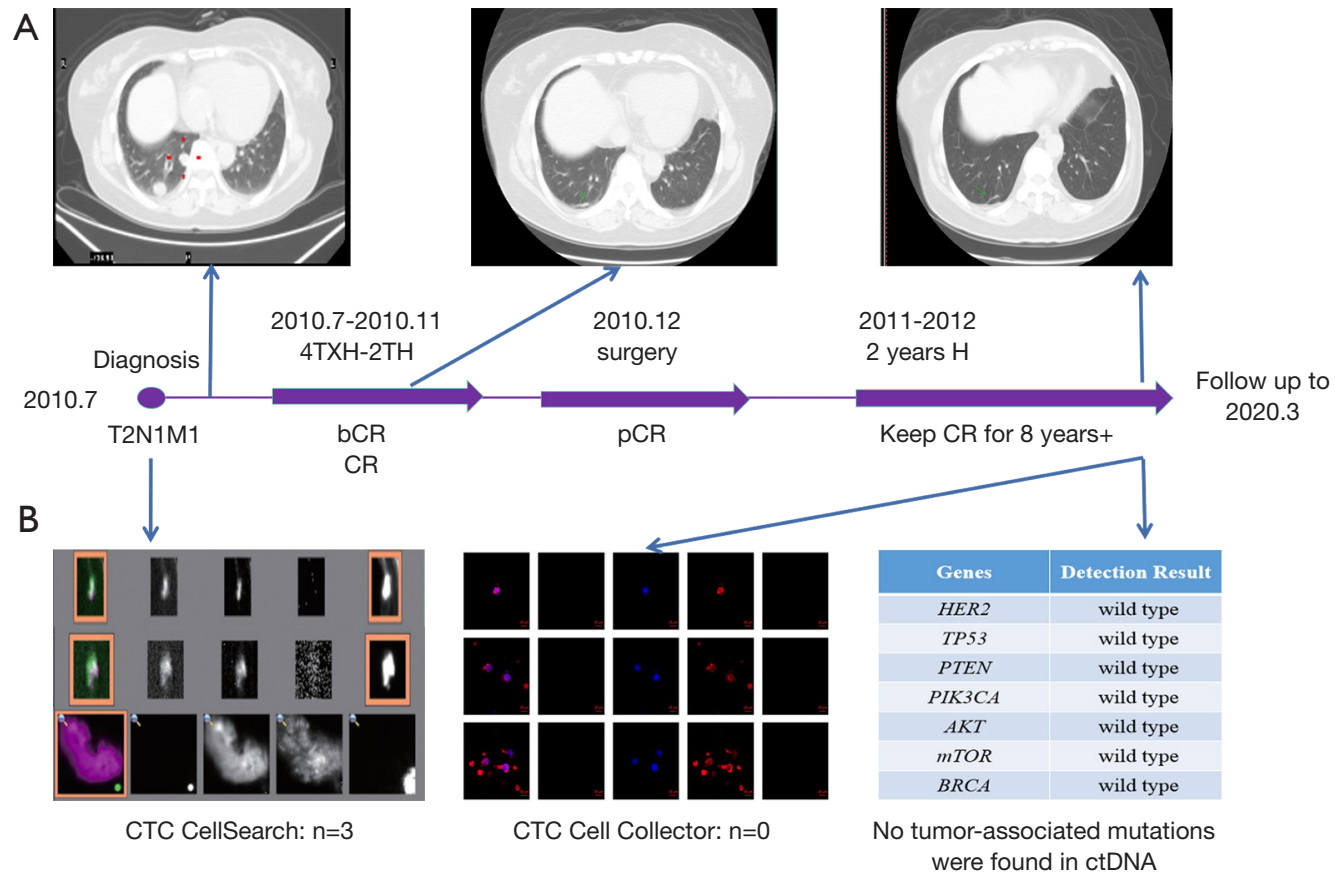

Figure 1 Patient treatment and examination results. (A) PET-CT scan images of lung metastasis; (B) detection of CTC and ctDNA. TXH, taxanes + capecitabine + trastuzumab; TH, taxanes + trastuzumab; CR, complete remission; bCR, biological complete remission; pCR, pathological complete remission; $\mathrm{H}$, trastuzumab; CTC, circulating tumor cell; ctDNA, circulating tumor DNA.

term disease-free survival benefit brought by bCR and the conversion of progression-free survival to disease-free survival in the patient with stage IV breast cancer. This case is reported in accordance with the CARE guidelines.

\section{Case presentation}

A 56-year-old woman presented in our department and was subsequently diagnosed with T2N1M1, stage IV cancer of the left breast, with metastasis to the left axillary lymph nodes and lungs. Immunohistochemistry showed ER(-), $\mathrm{PR}(-)$, and HER2(+++). After the diagnosis was confirmed, albumin-boundpaclitaxel (nab-paclitaxel, $200 \mathrm{mg}$, $126.6 \mathrm{mg} / \mathrm{m}^{2}, \mathrm{~d} 1$ and $\mathrm{d} 8 / 21$ days) and capecitabine (3,000 $\mathrm{mg}, 1,898.7 \mathrm{mg} / \mathrm{m}^{2}, \mathrm{~d} 1-14 / 21$ days) were used in combination with trastuzumab (440 mg, d1/21 days). Capecitabine was discontinued after four cycles, and paclitaxel plus trastuzumab were continued for two cycles. The patient experienced an adverse event in the form of a rash. After 1 cycle of treatment, the patient's CTC level decreased from 3 to 0 . After 3 cycles of treatment, the PET/CT SUVmax values of all lesions decreased to 0 , at which point "biological complete remission (bCR)" was achieved. After six cycles of therapy, the patient had complete remission (CR). Then, the patient underwent left breast mastectomy and left axillary lymph node dissection, and pathological complete remission (pCR) was confirmed. Subsequently, the patient received left chest wall and left clavicle radiotherapy, before 2 years of consolidation with trastuzumab. The patient has received no other treatment to date (Figure 1). The patient underwent regular medical examinations every year, and the results showed that she remained free from disease. The latest examination, in April 2019, by PET-CT showed no abnormal lesions (Figure 1A). CTC Cell Collector detected no circulating tumor cells, while before the initial treatment, the number of circulating tumor cells had been 3, as determined by the CTC Cell Search system. Meanwhile, the NGS test of ctDNA detected two unknown mutations of DNMT3A p.F752I and DAXX p.N633S, while the common tumor mutation test of the HER2 pathway was negative (Figure 1B).

\section{Discussion}

Maintenance therapy for advanced breast cancer after combination chemotherapy presents a number of options. 
In HER2-positive breast cancer, if the initial treatment is two types of chemotherapy combined with anti-HER2 targeted therapy, subsequent maintenance therapy may retain one form of chemotherapy combined with antiHER2 therapy. Meanwhile, trastuzumab is acceptable as a maintenance therapy for patients who cannot tolerate chemotherapy. After our patient had completed the regime of albumin-bound paclitaxel plus capecitabine combined with trastuzumab (TXH) for four cycles, we retained the treatment of TH for two cycles. After six cycles, complete remission of the breast lesion, axillary lymph nodes and the lung lesion had been achieved, and the patient received follow-up maintenance treatment of trastuzumab after surgery.

There is currently no recommendation for the duration of trastuzumab maintenance therapy, especially for patients with CR state after systemic therapy. The recommendations for multiple treatment vary between guidelines. The NCCN guidelines indicate that the duration of medication in patients with long-term control is undetermined. ASCO recommends that patients should continue with systemic therapy until disease progression or intolerable toxicity. The ABC-4 guidelines recommend that patients with complete remission for a long time may consider stopping treatment (3). Based on international consensus and treatment practices in China, the CSCO guidelines recommend that for patients who have achieved complete remission, the duration should be weighed against toxicity and the economic burden, and anti-HER2 treatment can be stopped after 2 to 3 years of remission. If the disease progresses at a later stage, anti-HER2 treatment can be resumed. For our patient, regular follow-up examinations showed that they had maintained a complete remission. We discontinued trastuzumab therapy in conjunction with the patient's wishes. The patient has so far maintained diseasefree survival, which shows that for patients with longterm control, especially those with complete remission, the suspension of the targeted therapy after a certain period of time is an acceptable option.

Liquid biopsy can explore tumor status in patients' peripheral circulation (4) and guide precision therapy (5). With the development of liquid biopsy, the technology of capturing CTC has continued to advance. CTC Cell Collector is a new detection technology that possesses higher sensitivity and specificity than CTC CellSearch (6). We performed detection with CTC Cell Collector on our patient, and the results showed that the number of CTCs was zero. Circulating tumor cells were still not detected using more sensitive detection methods, which suggested that the patient had complete tumor remission in the peripheral circulation.

Currently, ctDNA has application value in monitoring tumor burden, predicting drug efficacy, assessing risk of recurrence and metastasis, early diagnosis, and analyzing prognosis (7). The patient in this study was tested for 1,021 tumor-related genes, and only two-point mutations of $D N M T 3 A$ p.F752I and DAXXp.N633S were found. The two mutations of the MSK database were not recorded and their clinical significance is unknown. CTCs are generally recognized as tumor cells that have fallen off or migrated into the vasculature from the tumor lesions, and ctDNA is fragmented DNA in circulation from necrotic/apoptotic cells and/or is actively secreted from intact cells. Research on CTC and ctDNA has suggested that the ctDNA utilized in analysis may not originate from apoptotic/necrotic CTCs, but from actively shedding tumor cells (either in circulation or at primary/metastatic sites) (8). Therefore, the reason why CTC was not detected but ctDNA was detected in this patient is evident.

Studies have shown that mutations in the HER2 pathway in ctDNA may be related to resistance to antiHER2 treatment (9). In our patient, no related mutations were detected in the genes related to the HER2 pathway, including HER2, EGFR, ALK, and CDH1. It is also worth noting that in HER2-positive breast cancer patients, more than $60 \%$ of patients haveHER2amplification detected in ctDNA, but no HER2 amplification mutation was detected in ctDNA in the patient in this study. This maybe because most HER2-positive tumor cells are killed during longterm treatment. Both the negative HER2 amplification and negative HER2 pathway-related gene mutations indicate the stability of the HER2 pathway in this patient, which may also be interlinked with the long-term survival benefit observed.

Unlike early breast cancer, in which the primary survival indicator is disease-free survival, advanced breast cancer, due to the persistent presence of tumors in the body, is mainly evaluated based on progression-free survival. After receiving TXH treatment, the patient in this study achieved complete remission in imaging and pathology, and further CTC and ctDNA tests also indicated that complete remission had also been achieved in the peripheral circulation. For this patient, complete remission has continued for nearly 10 years. Although still in the advanced stage, the tumor status is closer to the tumor-free status. The patient's firstline therapy of PFS is closer to the DFS or EFS of early- 
stage patients in terms of treatment philosophy. For stage IV breast cancer patients, the initial rescue treatment is really the first-line treatment because no neoadjuvant and/ or adjuvant treatment has previously been received. The patient's first-line choices need to be optimized, and the conversion from progression-free survival to disease-free survival for stage IV patients should be pursued.

In this case report based on a real clinical case, we showed that a patient with stage IV breast cancer with distant metastasis, through optimized first-line therapy, could not only achieve progression-free survival, but also long-term disease-free survival. The rapid achievement of bCR after initial treatment may be the basis for obtaining long-term disease-free survival. Moreover, we analyzed the possible molecular mechanism from the level of CTC and ctDNA gene sequencing. The limitation of this case report is that the patient's CTC and ctDNA were not regularly detected during the follow-up period, and so any changes went unnoticed. In future research, we need to accumulate more similar patients in clinical practice to further explore the matter.

\section{Conclusions}

For advanced breast cancer, molecular detection technology can assist with our understanding of the molecular biological information of the disease. In this case, after TXH treatment, the patient's imaging, pathology and molecular biology results revealed complete remission. Two years of anti-HER2 targeted maintenance therapy not only ensured the maintenance of treatment efficacy to a certain extent, but also alleviated the patient's economic and psychological burden. The results of the patient's ctDNA, CTC, and PET-CT further confirmed that those with complete biological response to MBC may achieve long-term survival. Even for stage IV patients, active and reasonable systemic treatment can not only achieve progression-free survival, but also disease-free survival.

\section{Acknowledgments}

Funding: This study was supported by The National Natural Science Foundation of China [Grant number 81602314].

\section{Footnote}

Reporting Checklist: The authors have completed the CARE checklist (available at http://dx.doi.org/10.21037/ tbcr.2020.04.01).

Conflicts of Interest: All authors have completed the ICMJE uniform disclosure form (available at http://dx.doi. org/10.21037/tbcr.2020.04.01). ZJ serves as an unpaid Editor-in-Chief of Translational Breast Cancer Research. The other authors have no conflicts of interest to declare.

Ethical Statement: The authors are accountable for all aspects of the work including full data access, integrity of the data and the accuracy of the data analysis. The patient has given her consent for the case report to be published.

Open Access Statement: This is an Open Access article distributed in accordance with the Creative Commons Attribution-NonCommercial-NoDerivs 4.0 International License (CC BY-NC-ND 4.0), which permits the noncommercial replication and distribution of the article with the strict proviso that no changes or edits are made and the original work is properly cited (including links to both the formal publication through the relevant DOI and the license). See: https://creativecommons.org/licenses/by-nc-nd/4.0/.

\section{References}

1. Lane WO, Thomas SM, Blitzblau RC, et al. Surgical Resection of the Primary Tumor in Women With De Novo Stage IV Breast Cancer: Contemporary Practice Patterns and Survival Analysis. Ann Surg 2019;269:537-44.

2. Bian L, Wang T, Liu Y, et al. Evaluation of treatment response for breast cancer: are we entering the era of "biological complete remission"? Chin J Cancer Res 2012;24:403-7.

3. Cardoso F, Senkus E, Costa A, et al. 4th ESO-ESMO International Consensus Guidelines for Advanced Breast Cancer (ABC 4) dagger. Ann Oncol 2018;29:1634-57.

4. Crowley E, Di Nicolantonio F, Loupakis F, et al. Liquid biopsy: monitoring cancer-genetics in the blood. Nat Rev Clin Oncol 2013;10:472-84.

5. Xu F, Wang X, Jiang Z. The way to precision medicine of our team. Chin Clin Oncol 2016;5:46.

6. Budna-Tukan J, Swierczewska M, Mazel M, et al. Analysis of Circulating Tumor Cells in Patients with NonMetastatic High-Risk Prostate Cancer before and after Radiotherapy Using Three Different Enumeration Assays. Cancers (Basel) 2019;11:E802.

7. Romero D. Breast cancer: Tracking ctDNA to evaluate relapse risk. Nat Rev Clin Oncol 2015;12:624. 
8. Lowes LE, Bratman SV, Dittamore R, et al. Circulating Tumor Cells (CTC) and Cell-Free DNA (cfDNA) Workshop 2016: Scientific Opportunities and Logistics for Cancer Clinical Trial Incorporation. Int J Mol Sci 2016;17:E1505.

doi: $10.21037 /$ tbcr.2020.04.01

Cite this article as: Bian L, Xu F, Wang T, Zhang H, Cao Y, Jiang Z. Optimized first-line therapy for stage IV breast cancer brings long-term disease-free survival: a case report and biological analysis. Transl Breast Cancer Res 2020;1:7.
9. Ye Q, Qi F, Bian L, et al. Circulating-free DNA Mutation Associated with Response of Targeted Therapy in Human Epidermal Growth Factor Receptor 2-positive Metastatic Breast Cancer. Chin Med J (Engl) 2017;130:522-9. 